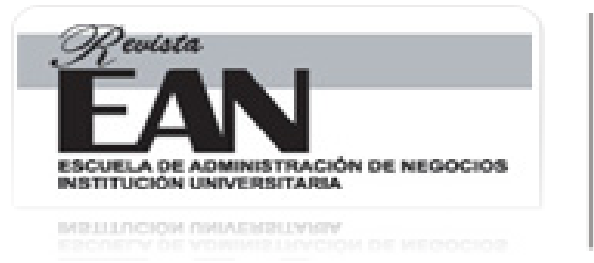

\author{
Revista Escuela de Administración de \\ Negocios \\ ISSN: 0120-8160 \\ investigaciones@ean.edu.co \\ Universidad EAN \\ Colombia
}

Páez, lliana; Rincón, Andrés; Astudillo, Margarita; Bohórquez, Stella

Un estudio de casos sobre liderazgo transformacional y competencias directivas en el sector floricultor de Colombia

Revista Escuela de Administración de Negocios, núm. 76, enero-junio, 2014, pp. 22-43

Universidad EAN

Bogóta, Colombia

Disponible en: http://www.redalyc.org/articulo.oa?id=20631321003

- Cómo citar el artículo

- Número completo

- Más información del artículo

- Página de la revista en redalyc.org

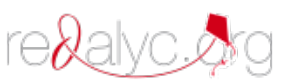

Sistema de Información Científica

Red de Revistas Científicas de América Latina, el Caribe, España y Portugal Proyecto académico sin fines de lucro, desarrollado bajo la iniciativa de acceso abierto 


\section{Un estudio de casos sobre liderazgo transformacional y competencias directivas en el sector floricultor de Colombia \\ (n)}

\author{
A Study of Transformational \\ Leadership and Managerial \\ Competencies in the Flawer \\ Agricultural Sector of Colombia
}

Etude de cas des politiques de transformation et des compétences de gestion dans le secteur de la flariculture en Calombie

Estudo de casa sobre liderança transformacional $e$ competências diretivas no setar flaricultar da Calômbia



Iliana Páez*

Andrés Rincón** Margarita Astudillo*** Stella Bohórquez ${ }^{* * *}$ 


\section{RESUMEN}

Un buen liderazgo es esencial para el desarrollo exitoso de las organizaciones y de sus miembros. El liderazgo transformacional, es un enfoque contemporáneo que resalta las bases morales del liderazgo y ha sido valorado en diversas culturas, asociándose con la efectividad del liderazgo y resultados en los trabajadores tales como satisfacción y desempeño. Se manifiesta a través de cuatro dimensiones básicas de comportamiento (influencia idealizada, motivación inspiradora, estímulo intelectual y consideración individualizada) y en las competencias de liderazgo de un dirigente. En este artículo, se analizan estas dimensiones $y$ diez competencias de liderazgo (integridad y confianza, orientación a la obtención de resultados, importancia en la acción, perseverancia, uso efectivo del tiempo, comunicación empática, conformación y desarrollo de equipos de alto desempeño, toma de decisiones, capacidad negociadora, y capacidad de direccionamiento estratégico), en los dirigentes de una muestra de 16 empresas del sector floricultor en Colombia con el sello Florverde.

Este sello, refleja el cuidado por la calidad de los productos y el compromiso de las empresas con el medioambiente y de responsabilidad social, aspectos que se asocian con las bases morales del liderazgo transformacional. Entre los directivos estudiados, se encontraron dos dimensiones de liderazgo transformacional: influencia idealizada y motivación inspiradora y su competencia más destacada fue la integridad y confianza, elementos claves para el desarrollo exitoso del liderazgo transformacional.

\section{ABSTRACT}

A good leadership is essential for the successful development of organizations and their staff members. Transformational leadership is a contemporary focus which highlights the moral bases of leadership and which has been valued in different cultures, being associated with its efectiviness and workers' outcomes such as satisfaction and performance. This leadership is manifested through four basic performance dimensions (idealized influence, inspiring motivation, intellectual stimulus and individual consideration) and through the managerial competencies of an executive. In this case study, these dimensions and ten leadership competencies (integrity and confidence, orientation to outcomes, importance of action, perseverance, effective use of time, effective communication, high performance team formation and development, decision making, business capacity and strategic management capacity) have been analyzed taking an executive sample of 16 companies from the flower agricultural sector with the Florverde seal.

This seal shows care for products and compromise with companies regarding environmental affairs and social responsibility, issues which are closely related with transformational leadership. Two transformational leadership dimensions were found: idealized influence and inspiring motivation, and its most outstanding competence was confidence and integrity, key elements for the successful development of transformational leadership.

\section{Palabras clave}

Liderazgo transformacional Liderazgo transaccional Competencias de liderazgo

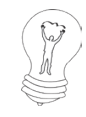

Transformational leadership Transactional leadership Leadership competencies

\section{Key words}






\section{RESUMÉÉ}

Un leadership efficace est essentiel au développement des organisations et de leurs membres. Le leadership transformationnel est une approche contemporaine qui met en évidence les fondements moraux de la direction d'entreprise et qui a longtemps été associé par différentes cultures à l'efficacité du comité de direction de l'entreprise. Au niveau des employés, cela se traduit par une plus grande satisfaction et une meilleure performance. Le leadership transformationnel se manifeste par le biais de quatre dimensions basées sur le comportement (influence idéalisée, source d'inspiration, de motivation et de stimulation intellectuelle) ainsi que sur les compétences de leadership d'un dirigeant. Cette étude de cas examine ces dimensions et dix compétences de leadership de dirigeants (intégrité et confiance axées sur les résultats, importance de l'action, de la persévérance, l'utilisation efficace du temps, l'empathie, la formation et les systèmes de communication de haut rendement, la capacité de décision, de négociation et stratégique) représentatif d'un échantillon de 16 entreprises du secteur floricole colombien estampillées Florverde.

Ce label reflète les soins apportés à la qualité des produits et à l'engagement des entreprises sur les questions environnementales et de responsabilité sociale. Ces aspects sont associés aux fondements moraux du leadership transformationnel. Deux dimensions de leadership transformationnel ont été mises en évidence chez les dirigeants participant à l'étude: l'influence idéalisée et la motivation inspiratrice. Les compétences les plus marquantes étant l'intégrité et la confiance en l'avenir, éléments clés de succès pour le développement du leadership transformationnel.

\section{RESUMO}

Uma boa liderança é fundamental para o desenvolvimento bem sucedido das organizações e seus membros. A liderança transformacional é um enfoque contemporâneo que ressalta as bases morais da liderança e foi valorizado em diversas culturas sendo associado à efetividade da liderança e aos resultados nos trabalhadores como satisfação e desempenho. A liderança transformacional é expressa através de quatro dimensões básicas de comportamento (influência idealizada, motivação inspiradora, estímulo intelectual e consideração individualizada) e nas competências de liderança de um dirigente. Neste estudo de caso analisam-se estas dimensões e dez competências de liderança (integridade e confiança, orientação à obtenção de resultados, importância na ação, perseverança, uso efetivo do tempo, comunicação empática, conformação e desenvolvimento de equipes de alto desempenho, toma de decisóes, capacidade negociadora e capacidade de direcionamento estratégico), nos dirigentes de uma amostra de 16 empresas do setor floricultor na Colômbia com selo Florverde.

Este selo reflete o cuidado pela qualidade dos produtos e o compromisso das empresas com assuntos ambientais e de responsabilidade social, aspectos associados às bases morais da liderança transformacional. Encontraram-se duas dimensões de liderança transformacional nos dirigentes estudados: influência idealizada e motivação inspiradora, as competências mais sobressalentes deles foram a integridade e confiança, elementos chaves para o desenvolvimento bem sucedido da liderança transformacional.

\section{Mots clefs}

\author{
Leadership \\ transformationnel \\ Leadership transactionnel \\ Compétences en \\ leadership
}

\section{Palavras-chave}

Liderança transformacional Liderança transacional Competências de liderança 


\section{INTRODUCCIÓN}

D e acuerdo con Yukl (2012), la esencia del liderazgo en las organizaciones tiene que ver con la forma en que los líderes influyen y facilitan los esfuerzos individuales y colectivos para lograr los objetivos. Es así como este se convierte en un factor clave para la gerencia, ya que de él dependen varios resultados importantes para las organizaciones, tales como desempeño individual y organizacional (Barling, Weber y Kelloway, 1996); satisfacción (Schriesheim, Castro, Zhou y DeChurch, 2006) motivación de los empleados (Bass, 1998; Judge y Piccolo, 2004); clima laboral (Holloway, 2012); y la misma supervivencia de las empresas (Barling, Weber y Kelloway, 1996; Rowe y Nejad, 2009).

Existen numerosas teorías que intentan explicar el éxito del liderazgo a partir de factores tales como los rasgos del líder, sus comportamientos, su interacción con el contexto y la situación, procesos de relaciones, procesos de poder e influencia, entre otros. Dentro de los enfoques contemporáneos, el liderazgo transformacional es un estilo ampliamente difundido que ha sido valorado positivamente a través de las culturas (Carl y Javidan, 2001), asociándose con la efectividad en el liderazgo (House, Javidan, Hanges y Dorfman, 2002) en dife-rentes países. Hablar de liderazgo transformacional es hablar de alinear intereses, no sólo de la organización, sino también de uno de sus factores esenciales: las personas. Es un estilo que está positivamente asociado con la satisfacción de los trabajadores, confianza en su líder y comportamientos ciudadanos en la organización (Podsakoff, MacKenzie, Moorman y Fetter, 1990). Las compañías con líderes transformacionales, suelen gozar de mayor descentralización de responsabilidad (Ling, Simsek, Lubatkin y Veiga, 2008), lo cual contribuye a aumentar el desempeño organizacional (Colbert, Kristof-Brown, Bradley y Barrik, 2008).

Sin embargo, los estudios sobre el rol del liderazgo transformacional en las empresas colombianas, son escasos. Por esta razón, es relevante conocer qué tanto se evidencia de este estilo en contextos cuyo desarrollo socioeconómico y características culturales difieren del entorno norteamericano, que es donde estas teorías han surgido y han sido más estudiadas, donde el interés por el desarrollo del otro ha sido un estilo tradicional inspirado por el modelo de Coach (entrenar y acompañar a otros) que utilizan los norteamericanos en entornos que van más allá de los escenarios deportivos. En Colombia este modelo no es tan común y además, las condiciones de desarrollo socioeconómico (más precarias que en Estados Unidos), hacen más retador para un dirigente adoptar el estilo transformacional cuando priman presiones por resultados inmediatos de carácter netamente económico. De esta manera, se espera contribuir al conocimiento teórico sobre la forma como se manifiestan las dimensiones del liderazgo transformacional en el contexto colombiano, e implicaciones prácticas sobre qué factores potencian el desarrollo de este estilo en los directivos estudiados.

El sector floricultor, es un sector de larga tradición en Colombia que ha mostrado ser exitoso por más de una década consecutiva, ocupando los primeros tres puertos de exportación de flores en el mundo. Es un sector que además de generar empleo en poblaciones vulnerables, se ha preocupado por atender las necesidades sociales y ambientales más allá de lo exigido por la ley, donde cada vez más empresas del sector obtienen el sello de Florverde, que expresa el compromiso de Asocolflores de apoyar y exigir un alto compromiso de responsabilidad social y ambiental en estas empresas. Adicionalmente, dado que el liderazgo se manifiesta, percibe y evalúa a través de las competencias que demuestre el líder en su rol, es pertinente identificar qué competencias de liderazgo muestran los líderes en las empresas colombianas y en qué medida se asocian con el liderazgo transformacional. Estas características sugieren sensibilidad por el otro y valores morales por parte de los dirigentes de estas empresas, características que se relacionan con los postulados del liderazgo transformacional. 
El propósito de este artículo, es analizar las características de liderazgo transformacional y las competencias de liderazgo que presentan los gerentes del sector floricultor en Colombia, con el ánimo de identificar sus fortalezas en términos de las dimensiones de liderazgo transformacional que puedan servir para mejorar la capacidad directiva de estos gerentes. Está compuesto por cuatro secciones. En la primera, se presenta el marco conceptual utilizado que incluye los fundamentos teóricos sobre liderazgo y los estilos transaccional y transformacional, así como sobre competencias directivas. En la segunda, se describen los aspectos metodológicos del estudio realizado con líderes de empresas del sector floricultor. En la tercera, se resumen los resultados obtenidos con relación a los comportamientos de liderazgo transaccional, las dimensiones de liderazgo transformacional y las competencias de liderazgo identificadas en los líderes de las empresas del sector floricultor. Finalmente, se presentan las conclusiones del estudio, don-de se resumen los principales hallazgos y se indican caminos de profundización e investigación futura.

\section{FUNDAMENTO TEÓRICO}

\subsection{Liderazgo}

Los estudiosos del liderazgo se han preocupado por identificar los factores que lo hacen efectivo en las organizaciones. En las últimas décadas (treinta y posteriormente en los noventa), estos factores se han analizado desde los rasgos del líder; luego, desde sus comportamientos (en la década de los cincuenta) y desde los procesos de poder e influencia (en la década de los sesenta); más adelante, se tuvieron en cuenta los factores situacionales y de contingencia (en la década de los setenta); posteriormente, surgieron una serie de enfoques que integraban varios aspectos de los anteriores pero desde una perspectiva más humana donde se resalta la importancia de las relaciones, los valores y la dimensión ética del liderazgo, creando una generación de enfoques contemporáneos que sigue evolucionando hasta el día de hoy (Yammarino, Dionne, Chun y Dansereau, 2005; Yukl y Van Fleet, 1992).

Desde la perspectiva tradicional, el liderazgo era entendido como un proceso de influencia sobre personas, procesos y objetivos (Yukl y van Fleet, 1992). Los enfoques contemporáneos, por su parte, hacen énfasis en la capacidad que tiene el líder de generar confianza (Dirks y Ferrin, 2002), inspirar a los demás (Bass, 1985), de ser auténtico (Walumbwa, Avolio,
Gardner, Wernsing, y Peterson, 2008), de relacionarse con sus colaboradores (Liden, Sparrowe y Wayne, 1997) y de comportarse y ser un modelo de rol en conducta ética (Brown, Treviño y Harrison, 2005).

Desde los enfoques contemporáneos, se empiezan a ser evidentes los aspectos morales del liderazgo (Ciulla, 1998). Según Knights y O'Leary (2006), la sola existencia de otros, hace a los líderes moralmente responsables hacia estos. El liderazgo entonces, es visto como un fenómeno relacional (Graen y Uhl-Bien, 1995) donde se reconoce de una manera más explícita que en el proceso de influencia, los líderes pueden afectar a las personas con las que

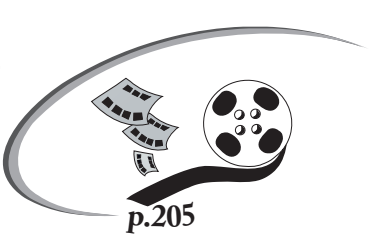
se relacionan (Gini, 1998). Por lo tanto, dado que el liderazgo abarca diferentes relaciones humanas con diferentes problemas morales (Ciulla, 1998), el proceso de influencia en el liderazgo debe ejercerse con responsabilidad (Cortina, comunicación personal 2008) y respetando la dignidad del otro. Es así, como la ética y las competencias de liderazgo se vuelven centrales en el desempeño de los líderes (Kort, 2008). Dentro de esta perspectiva, Páez, González, Enciso, Perilla y Martínez (2012), conciben el liderazgo como la capacidad de 
guiar a las personas hacia un propósito colectivo, desde un proceso de influencia inspirador basado en la autenticidad y los valores morales, procurando su crecimiento personal, mientras se protegen sus intereses individuales.

El liderazgo transformacional, es un enfoque contemporáneo que resalta las bases morales del liderazgo, porque tiene en cuenta los valores del líder y el reconocimiento explícito del otro (Bass y Steidlmeier, 1999). Surge a finales de la década de los setenta como una propuesta para mejorar la efectividad del liderazgo tradicional, conocido como transaccional, que se basaba en las transacciones entre un líder y sus seguidores. El liderazgo transformacional, empezó a ser victo entonces como un complemento al liderazgo transaccional al enfocarse en el desarrollo de los trabajadores. Según Bass (1985), el liderazgo transaccional es un prerrequisito clave para el liderazgo efectivo. Cuando esto se da es cuando realmente se potencializa la capacidad y desempeño de los trabajadores.

\subsection{Liderazgo transaccional}

En el liderazgo transaccional, el líder guía a sus colaboradores hacia metas establecidas mediante requisitos específicos y roles bien definidos, donde los éxitos se premian y los fracasos se castigan (Antonakis y House, 2002); en otras palabras, es un enfoque centrado llanamente en los resultados. Este enfoque abarca cuatro estilos básicos que reflejan diferentes tipos de intervención del líder y son: política de no intervención, conocido como Laissez-Faire, en la que el líder evita las responsabilidades y la toma de decisiones (Eagly, Johannesen-Schmidt y Van Engen, 2003); le sigue gerencia por excepción pasiva, en la que el líder interviene sólo cuando no se cumplen los estándares; luego está la gerencia por excepción activa, en la que el líder acompaña el proceso haciendo correctivos oportunamente; y finalmente, recompensa contingente, en la que el líder reconoce los logros y los recompensa.

Los dos primeros comportamientos del estilo de liderazgo transaccional se conocen como no liderazgo y están caracterizados por ejercerse en una gerencia pasiva que ha demostrado ser inefectiva (Hinkin y Schriescheim, 2008). Los dos siguientes comportamientos del liderazgo transaccional son más efectivos (Lowe, Kroeck y Sivasubramaniam, 1996) y se caracterizan por una intervención más activa del líder sobre su grupo y los procesos que lidera. En la gerencia por excepción activa, el líder da libertad de acción a sus colaboradores, sin embargo toma correctivos apenas detecta errores o desviaciones de las reglas y estándares definidos previamente. Finalmente, con la recompensa contingente, el líder define claramente los requerimientos de rol y tarea, reconoce logros y ofrece recompensas contingentes al desempeño (Antonakis y House, 2002).

En resumen, el liderazgo transaccional es un estilo que contribuye a la clarificación y cumplimiento de metas, pero prácticamente descuida el crecimiento y los intereses de los colaboradores en pro de esas metas. Sin embargo, el liderazgo transaccional sienta unas bases sólidas y prácticas sobre las que se puede desarrollar el liderazgo transformacional.

\subsection{Liderazgo transformacional}

El liderazgo transformacional, se caracteriza por la capacidad del líder para inspirar a sus colaboradores a que trasciendan sus intereses individuales hacia metas colectivas (Bass, 1985), a la vez que los motiva a desempeñarse mejor y fomenta su desarrollo personal. Se caracteriza por el reconocimiento al otro, así como por la preocupación genuina del líder por el crecimiento de sus colaboradores (Bass y Steidlmeier, 1999). Se concibe como la suma de dos esfuerzos: el del líder y el de los colaboradores, que se conjugan en pro de un bien colectivo.


El líder se convierte en el guía inspirador y en un modelo de rol para sus colaboradores, generando cambios en las actitudes de quienes lo siguen sin descuidar su propio actuar.

Bass (1985), propuso que el liderazgo transformacional es complementario al transaccional y cuando el primero se construye a partir del segundo, se producen 
resultados extraordinarios tales como esfuerzos y desempeños superiores a lo que se logra sólo con el liderazgo transaccional. Según Burns (1978), mientras que desde el liderazgo transaccional los líderes se enfocan en intercambios con sus seguidores teniendo en cuenta sus necesidades actuales, desde el liderazgo transformacional los líderes buscan elevar las necesidades de sus colaboradores y promueven cambios significativos a nivel individual y organizacional. De acuerdo con Bass, el líder transformacional inspira y motiva a sus colaboradores a partir de crear visiones realistas que puedan ser compartidas, los estimula intelectualmente y tiene en cuenta sus diferencias individuales.

Los resultados del liderazgo transformacional han mostrado ser muy positivos, tanto para la organización como para las personas que laboran en ella. Los estudios han mostrado que el liderazgo transformacional se relaciona con niveles más altos de desempeño individual de los colaboradores, desempeño grupal y organizacional (Wang, Oh, Courtright y Colbert, 2011). Los líderes transformacionales fomentan en sus colaboradores un desempeño superior (Bass, 1999), porque tienen la capacidad de aumentar en ellos el sentido de autoeficacia (Walumbwa, Avolio y Zhu, 2008), fomentan la creatividad (Shin y Zhou, 2003; Zhang y Bartol, 2010), y generan compromiso (Avolio, Zhu, Koh, y Bathia, 2004; Dvir, Kass y Shamir, 2004) y bienestar (Liu, Siu y Shi, 2010).

Los líderes transformacionales, influyen positivamente en resultados grupales porque mejoran el clima de trabajo en equipo y promueven la innovación al fomentar el consenso, integrar valores y crean un sentido de propósito elevado (Eisenbeiss, Van Knippenberg y Boerner, 2008; Schaubroeck, Lam y Cha, 2007; Zohar y Tenne-Gazit, 2008). También, se ha encontrado que el liderazgo transformacional aumenta el desempeño y la innovación a nivel organizacional, porque los líderes logran que sus colaboradores asuman las metas organizacionales como algo personalmente importante (Berson y Avolio, 2004; Bono y Judge, 2003; Colbert et al., 2008); García-Morales, Lloréns-Montes y VerdúJover, 2008).
Para que el liderazgo transformacional sea efectivo, el líder debe mostrar y encarnar un conjunto de comportamientos específicos conocidos como dimensiones del liderazgo transformacional. Bass (1998), formuló que este liderazgo ejerce su influencia a través de cuatro dimensiones: influencia idealizada, motivación inspiradora, estímulo intelectual y consideración individualizada.

Influencia idealizada. Hace referencia a qué tanto el seguidor se identifica e intenta emular al líder, ya que este último puede, por medio de su carisma fundamentado en valores legítimos morales, inspirar a realizar más de lo que dicta el deber, el líder antepone las necesidades de los demás a las suyas propias (Bass, 1998). El carisma, como también se le conoce a la influencia idealizada, suele reflejar intenciones altruistas por parte de un líder transformacional, que procura alcanzarlas de manera legítima sin recurrir a la dependencia que genera la idolatría (Barling, Christie, Turner 2008). Según Shamir, House y Arthur (1993), cuando el líder logra que sus seguidores se sientan más identificados por la misión y los objetivos propuestos, dichos seguidores comienzan a experimentar mayor compromiso, los grupos son más cohesivos, se involucran más, lo que da lugar a un incremento en el desempeño.

Motivación inspiradora. Es la capacidad del líder para inspirar y motivar a sus colaboradores, para alcanzar una visión fuerte del futuro. Para esto, el líder busca apelar a lo mejor de las personas dando lugar a que le encuentren sentido y significado a su labor; así mismo, logra que participen en la consecución de metas colectivas (Bass, 1998). Mediante esta dimensión, los líderes comunican altas expectativas y articulan visiones retadoras, alcanzables y esperanzadoras. Según Avolio, Walumbwa y Weber (2009), el desempeño y el compromiso de los seguidores de un líder transformacional, pueden trascender su egoísmo y expectativas individuales.

Estímulo intelectual. Se refiere a la forma e intensidad con la que el líder busca aumentar la capacidad creativa de sus colaboradores, fomentando la generación de nuevas ideas y el cuestionamiento de supuestos existentes (Bass, 1998). Según Bandura (1986), cuando los líderes estimulan intelectualmente a sus seguidores, 
la creatividad aumenta, pues estos toman el papel de guías que establecen desafíos para los trabajadores, lo que funciona conjuntamente con la habilidad del líder para que el trabajador pierda el miedo de desafiar lo establecido.

Consideración individualizada. Consiste en que el líder se interesa por sus colaboradores como personas. En este sentido, esta dimensión refleja la capacidad del líder de reconocer y atender las necesidades individuales de sus colaboradores. El líder actúa como un mentor, con el fin de otorgar oportunidades de crecimiento personalizadas, cumpliendo así una doble función: inspirar a sus colaboradores y transformarlos en líderes (Bass, 1998). En palabras de Burns (1978), por medio de la escucha atenta a las aspiraciones, necesidades y habilidades, el líder toma el rol de consejero, profesor y entrenador con el fin de contribuir con el crecimiento de los trabajadores.

Cuando dichas dimensiones comienzan a actuar de manera concertada, los beneficios se perfilan con mayor intensidad, pues ello hace parte del carisma y de la capacidad de inspirar del líder. Investigadores como Bass (1985), Dvir y colegas (2004), concluyen que bajo el liderazgo transformacional, los trabajadores tienden a estar más dispuestos a escuchar y a aprender de sus líderes.

Además de estas dimensiones de comportamiento del liderazgo transformacional, el liderazgo de los dirigentes se evidencia a través de sus competencias directivas. Estas competencias son un saber hacer en contexto, que dentro del marco del presente estudio, abarcan las competencias de liderazgo que hacen que un dirigente sea efectivo en su rol gerencial.

\subsection{Las competencias del dirigente- líder}

El Centro de Liderazgo de la Facultad de Administración de Empresas ${ }^{1}$, identificó diez competencias asociadas al liderazgo en la gerencia (Páez, Maldonado y Murillo, 2004) y elaboró un marco conceptual sobre estas, desde una perspectiva ética que las integra con el estilo de liderazgo transformacional (Páez et al., 2012). Estas son: integridad y confianza, orientación a la obtención de resultados, importancia en la acción, perseverancia, uso efectivo del tiempo, comunicación empática, conformación y desarrollo de equipos de alto desempeño, toma de decisiones, capacidad negociadora y capacidad de direccionamiento estratégico.

Integridad y confianza. Es la primera competencia y quizás la más compleja, ya que describe el obrar del líder, según el cual este es coherente, auténtico y correcto en su actuar. Se define como la "capacidad de obrar con honestidad y rectitud y de involucrarse en comportamientos moralmente correctos, demostrando coherencia entre lo que se dice y lo que se hace, generando confiabilidad". La confiabilidad a su vez, es "la capacidad de generar confianza en los demás quienes sienten que no van a ser defraudados. Tener integridad y confianza, es obrar con rectitud y coherencia mostrando adherencia a valores morales" (Páez, 2012, p90). Cuando los líderes organizacionales buscan ganar credibilidad en los trabajadores, la integridad se vuelve un factor decisivo (Simons, 1999), ya que les permite generar confianza en el líder como jefe y como ser humano. De hecho, se ha encontrado un vínculo fuerte entre la integridad y aquellos factores que son clave en un proceso de cambio, como lo es la confianza y el compromiso. Por el contrario, de acuerdo con Simons, la ausencia de un buen obrar por parte del líder puede derivar en una menor capacidad de

\footnotetext{
1 El Centro de Liderazgo, es un centro de investigación y desarrollo en el tema de liderazgo y sus competencias. Pertenece a la Facultad de Administración de Empresas de la Universidad Externado de Colombia, creado en 2001 para desarrollar proyectos de investigación, definir los contenidos y guías metodológicas para los programas académicos y ofrecer materias, seminarios y programas dentro de la Universidad y para la comunidad empresarial y social.
} 
persuasión. La integridad y confianza se constituye en la competencia central del líder transformacional, a partir de la cual el líder es percibido como alguien confiable, atributo al que mejor responden los colaboradores (Dirks y Ferrin, 2002).

Orientación a la obtención de resultado. Esta segunda competencia, se relaciona con la capacidad del gerente para llevar a cabo la misión y visión de la empresa, lo cual, desde la perspectiva de liderazgo transformacional, debe realizarse integrando los esfuerzos individuales y colectivos. Se define como la "capacidad de plantearse metas y focalizarse decididamente en los objetivos, reconociendo los efectos y las implicaciones derivadas de las acciones emprendidas, privilegiando aquellas consideradas como más convenientes para la organización en la consecución y logro de los resultados, aun en condiciones adversas" (Páez et al., 2012, p110). Los líderes más capaces, buscan integrar un acercamiento que incluya tanto la orientación a la obtención de resultados, como una orientación hacia las relaciones (Blake y Mouton, 1982). Es así, como el líder transformacional gestiona diversos aspectos para llegar a resultados concretos por medio de su capacidad técnica y sensibilidad humana, lo que da lugar a que se estimule a los trabajadores a realizar más de lo que se espera de ellos (Burns, 1978), elevando a su vez, el desempeño colectivo. Los estudios sobre las consecuencias del liderazgo transformacional han mostrado que existe una relación positiva con el desempeño individual y grupal (Lowe. et al., 1996).

Importancia en la acción. Esta competencia hace referencia a la capacidad del líder de convertir en acciones aquellas ideas que se generan en el día a día sin que sea una necesidad implícita los largos procesos de planeación. Se define como la "capacidad de actuar de manera intensa y con energía, a partir de una intención clara, identificando, aprovechando y generando oportunidades y pudiendo convertir en acciones concretas, las ideas, los planteamientos y los proyectos, sin requerir largos y extenuantes periodos de planeación" (Páez et al., 2012, p.125). Sin embargo no es un aspecto únicamente de ejecución, es también un aspecto de saber el momento preciso para actuar, pues actuar de manera reactiva y tardía ha mostrado ser poco efectivo en el liderazgo en términos de motivación o desempeño de una unidad e trabajo (Bass, Avolio, Jung y Berson, 2003).

Perseverancia. Según Bass (1985), el liderazgo transformacional logra que los grupos persistan cuando las condiciones son adversas, donde prima lo impredecible y lo difícil. La perseverancia se relaciona a la manera como los líderes desarrollan los objetivos y las metas establecidas sin desfallecer. Se define como la "capacidad de mantenerse firme y constante en la consecución de propósitos, objetivos y metas, y en el desarrollo de las acciones en ellos involucradas. Implica una fuerza interior, constituida por elementos racionales, emocionales y volitivos, que permiten insistir de manera reflexiva, superando obstáculos, neutralizando las dificultades y las oposiciones" (Páez et al., 2012, p125). La perseverancia suele verse reflejada en las organizaciones con líderes transformacionales, cuando estos retan a sus seguidores a pensar de forma diferente, ya que dicho comportamiento estimula el sentimiento de lograr más allá de lo que se creía posible.

Uso efectivo del tiempo. Esta competencia hace referencia a la forma como el líder emplea su tiempo y su capacidad para realizar sus tareas y funciones de manera oportuna. Es definida como el equilibrio entre la vida intelectiva, emocional y volitiva del Dirigente-Líder en los diferentes roles de su vida profesional y personal (Páez et al., 2012). Requiere saber identificar prioridades en los diferentes ámbitos y actuar sobre ellas en pro de cumplir con las metas organizacionales sin perder el verdadero sentido de vida para sí mismo y para los demás. Según Álvarez de Mon y colegas (2001), para un uso efectivo del tiempo es fundamental diferenciar la vida personal, familiar y profesional con el fin de separar lo que es importante de lo que es urgente.

Comunicación. Esta competencia no solo se refiera a la capacidad para transmitir información clara y oportuna de manera asertiva y respetuosa, sino también la capacidad de escucha del líder. Se define como la "capacidad para escuchar activamente a otros y expresarse de manera empática, asertiva y dentro de un marco de respeto que permite compartir ideas, sentimientos e información de forma clara, honesta y oportuna, para influir, persuadir y en general, transmitir correctamente significados, cuya 
verificación se realiza a través de una adecuada retroalimentación" (Páez et al., 2012, p185). Cuando los líderes se comunican de manera efectiva y congruente, ya sea compartiendo una visión amplia que logra que los seguidores vean los objetivos organizacionales importantes, o resaltando consistencias entre el trabajo realizado por sus seguidores y el de la organización, logran que sus unidades de trabajo sean más efectivas (Hoffman, Bynum, Piccolo y Sutton, 2011). Los líderes transformacionales se comunican de manera honesta y transparente porque tienen en cuenta el otro y lo respetan como individuo y como parte de una colectividad.

\section{Conformación y desarrollo de equipos de alto desem-} peño. Es todo lo referente a cómo el líder utiliza su capacidad para conformar y potenciar a sus colaboradores individualmente y como equipo a favor de metas colectivas. Se define como la "capacidad mediante la cual el Dirigente-Líder selecciona los miembros del equipo de acuerdo con las competencias requeridas en el proyecto a realizar, comunica los objetivos y metas estableciendo estándares de ejecución retadores, motiva a los miembros para que los alcancen o sobrepasen trabajando con disciplina, sinergia y responsabilidad compartida, promoviendo el desarrollo de los miembros del equipo, ofreciendo desafíos que les permitan descubrir y afianzar su potencial, en un ambiente positivo de trabajo" (Páez et al., 2012, p206). En la literatura se encuentran ambigüedades respecto al liderazgo de los equipos, ya que por un lado se dice que el desempeño inicial de los equipos empoderados es eclipsado por aquellos equipos regidos por liderazgo directivo, sin embargo, con el tiempo, los papeles se invierten, pues el aprendizaje que se da al interior de los grupos permite que aumente la coordinación (Lorinkova, Pearsall, y Sims Jr., 2013). Según Carson, Tesluk y Marrone (2007), la clave del buen desempeño de los equipos reside en compartir el liderazgo. Dado que el liderazgo transformacional promueve el desarrollo de los seguidores, es más probable que el proceso del equipo se desarrolle de manera más favorable. Según LePine, Piccolo, Jackson, Mathieu y Saul (2008), el proceso del trabajo en equipo se relaciona positivamente con el desempeño del equipo y la satisfacción de sus miembros.
Toma de decisiones. Hace referencia a la habilidad que tiene el líder para tomar decisiones de manera acertada y oportuna. Se define como la capacidad de identificar, formular e implantar una o más soluciones a los problemas desde procesos de análisis, síntesis y evaluación, a la vez que minimiza riesgos para la consecución de objetivos y metas (Páez et al., 2012, p232). Los líderes transformacionales, tienen en cuenta las consecuencias de sus actos cuando toman decisiones. Se guían por valores éticos que buscan proteger la integridad de sus colaboradores y personas que puedan ser afectadas por sus decisiones. Según Messick y Bazerman (1996), la toma de decisiones sistemática y concreta, contribuye a asegurar la calidad del resultado, y a evitar errores éticos.

Capacidad negociadora. Se refiere a la capacidad para persuadir a otros en beneficios mutuos y bajo un marco ético. Se define como la "capacidad para utilizar estrategias, tácticas y argumentos sólidos para persuadir a otros con un marco ético y llegar a acuerdos que favorezcan los intereses, deseos y necesidades comunes a las partes, creando un ambiente propicio para la colaboración y logrando relaciones beneficiosas y compromisos duraderos" (Páez et al., 2012, p252). El líder transformacional, actúa bajo un marco ético porque el fundamento de este estilo está en valores morales que guían al líder y lo convierten en un modelo de rol para los demás. Según Lewicki, Saunsers y Minton (1999), cuando los líderes examinan los aspectos éticos en sus procesos de negociación, hace que analicen mejor sus estrategias y decisiones y logren mejores relaciones a largo plazo. Luecke (2003), recomienda un acercamiento desde la cooperación entre las partes con el fin de maximizar los beneficios con base en la integración de intereses para dar forma a un acuerdo, incluso si dichos intereses entre las partes no son convergentes.

Capacidad de direccionamiento estratégico. Esta competencia consiste en la manera como el líder idea, e implementa estrategias con el fin de lograr los objetivos propuestos con los resultados esperados. Se define como la "capacidad de formular e implementar estrategias que le permitan dirigir la organización hacia el éxito, basadas en el conocimiento e interpretación de los cambios del entorno y su impacto en el ámbito de la organización, con 
una amplia visión de futuro y múltiples perspectivas que favorezcan a todos los Stakeholders" (Páez et al., 2012, p268). Un aspecto clave para guiar a la organización en la dirección hacia el éxito, tiene que ver con qué tan efectivo es el líder para comunicarse, pues es así como el líder puede dar a conocer y lograr que se interioricen los objetivos organizacionales estratégicos, por parte de sus seguidores (Berson y Avolio, 2004). También está presente un fuerte componente de comprender el medio donde se mueve la empresa, pues es así como identificando oportunidades y amenazas, se establece una visión amplia para la organización (Yukl, 2012).
Las competencias descritas, tienen un fuerte sentido moral y ético, definen la base sobre la que se construye un líder transformador efectivo, capaz de dar lugar a desarrollos positivos que impactan a las organizaciones, así como a las personas que las constituyen. A continuación, se expondrá la metodología empleada para recopilar la información de las diversas empresas del sector floricultor en el país que fue determinante para el desarrollo de este artículo.

\section{METODOLOGÍA}

$\mathrm{E}$ ste artículo se basó en entrevistas y grupos focales con el personal administrativo y trabajadores de 16 empresas del sector floricultor en Colombia, que pertenecen al gremio de Asocoflores ${ }^{2}$ y que han sido certificadas con el sello de Florverde ${ }^{3}$. Estos aspectos aseguran que las labores de floricultura llevadas a cabo por dichas organizaciones sean competitivas y sostenibles en diversos ámbitos y con un claro enfoque social y ambiental. Se seleccionaron empresas con estas características porque todo esto sugiere la presencia de bases de liderazgo transformacional ya que se demuestra un claro interés de sus directivos por ir más allá de lo convencional, así como una notoria sensibilidad por hacer las cosas bien interesándose por el otro (responsabilidad social) y protegiendo los intereses de diferentes Stakeholders (medio ambiente, por ejemplo).

El papel del sector floricultor en la economía de Colombia, ha sido destacado en las últimas tres décadas, especialmente como un estandarte de los productos del país en mercados internacionales. Asocolflores, el gremio de dicho sector, estima que el $98 \%$ de la producción se destina a satisfacer la demanda de mercados internacionales, especialmente el mercado doméstico de Estados Unidos y otros más distantes como el mercado japonés. Se escogió este sector por el importante papel que ha venido representando en la economía nacional en los últimos 35 años ocupando los primeros puestos de exportaciones de flores en el mundo y aportando el 4\% del PIB Agrícola Nacional (Asocolflores); también porque el sello Florverde demuestra que los dirigentes de este sector, se preocupan por aspectos que reflejan principios morales tales como garantizar la calidad de sus productos, proteger el medio ambiente y ser socialmente responsables. Aunque resulta evidente que el liderazgo transformacional, como estilo de gestión va mucho más allá de los aspectos que sugiere el sello Florverde, consideramos que es el estilo que más concuerda con esta directriz y esperamos se vea reflejado en el estudio. El liderazgo transformacional tiene bases morales claras debido a que su constructo incluye explícitamente la preocupación por el bienestar del otro y la generación elevada de propósitos, que dan mayor sentido a su labor y la de sus colaboradores, a la vez que respeta su dignidad y promueve su desarrollo.

\footnotetext{
2 Asociación Colombiana de Exportadores de Flores, organización gremial sin ánimo de lucro que representa floricultores del país.

3 Instrumento que busca promover la floricultura sostenible, define estándares sociales y ambientales en todo el proceso productivo del sector floricultor.
} 
Esta fue razón para querer conocer más sobre la cultura empresarial de dicho sector, lo que permitiría determinar el papel del liderazgo en la muestra estudiada. Con esto, se busca identificar y describir las características de los gerentes en términos del liderazgo transformacional y las diez competencias de liderazgo.

La metodología utilizada, incluye una entrevista a profundidad al gerente (categorías: historia de vida, rasgos de personalidad, valores e intereses, factores de éxito, cualidades de liderazgo general, inspiraciones, motivaciones, estímulo y estilo de vida); dos grupos focales, uno con los colaboradores directos del gerente y otro con el personal operativo (características de liderazgo del gerente, factores de éxito y tipo de relación); una encuesta al gerente sobre su identificación y valoración de cada una de las diez competencias de liderazgo ordenándolas de uno a diez, siendo diez la más importante y representativa y uno la menos importante y representativa en su gestión y estilo de liderazgo.

El método seguido fue el de análisis de contenido, con el objeto de identificar alusiones o respuestas confirmatorias sobre comportamientos característicos en las cuatro dimensiones de liderazgo transformacional, así como el ordenamiento jerárquico de las diez competencias de liderazgo.
Las entrevistas a profundidad se realizaron únicamente a los altos directivos de las 16 empresas consultadas, que incluyen gerentes de área, vicepresidentes y presidentes. Estas, buscaban determinar el perfil profesional, así como los rasgos, valores e intereses que los definen para posteriormente indagar sobre sus factores de éxito y aquello que los convertía en líderes y lo que definía su estilo de liderazgo, pues sería determinante para establecer el rol del liderazgo transformacional en el sector.

Por su parte, los grupos focales se concentraron en los trabajadores, tanto de nivel administrativo como de nivel operativo, buscando descubrir qué los caracteriza, su concepción de liderazgo, así como sus opiniones sobre aquellos que dirigen su área o la organización, específicamente si los consideran líderes por convicción o si lo hacen únicamente por razones de autoridad y cómo ejercen su liderazgo (estilo y forma de relacionarse con sus colaboradores y clientes).

Finalmente, con base en la información recopilada, se identificaron diversos líderes informales, los cuales se entrevistaron a profundidad buscando indagar lo mismo que se buscó en los altos directivos consultados, pues ello permitiría determinar con mayor precisión, de qué forma se manifiesta el liderazgo en el sector floricultor colombiano.






\section{RESULTADOS}

L as entrevistas realizadas a los directivos de las empresas, permitieron determinar que el $100 \%$ son profesionales, lo que evidencia que es un requisito indispensable para alcanzar los cargos gerenciales. El 10\% de las personas entrevistadas tienen estudios adicionales al pregrado, lo que sugiere que son fomentados por interés propio más que por necesidades de la empresa o de sus funciones. Los dirigentes entrevistados, son todos mayores de 30 años y ninguno está por encima de los 58 años, la gran mayoría (63\%) está entre los 30 y 42 años.

La distribución de géneros es bastante pareja, aunque tiene una ligera inclinación hacia los hombres, representados en un $56 \%$, lo que demuestra que no se presenta discriminación de género en el sector. Tanto hombres como mujeres coincidieron en que un factor clave para el éxito, era destacarse académicamente, pues dichas entrevistas permitieron entrever que no se presentan fenómenos de clientelismo. Por otro lado, afirmaron que para ascender en la escalera empresarial lo que importa es la trayectoria al interior de la organización, así como los logros y características individuales. Finalmente, resulta claro que en su totalidad son personas trabajadoras, pues cumplen con horarios laborales extensos de al menos 12 horas, lo que los obliga a sacrificar tiempo para otras actividades, aunque no quiere decir que haya total ausencia de ellas.

A continuación, se presentan los resultados del estudio con relación a los tres aspectos centrales de este artículo: comportamientos de liderazgo transaccional, comportamientos y dimensiones de liderazgo transformacional y finalmente, la presencia y características de las diez competencias de liderazgo en los líderes del sector floricultor.

\subsection{Comportamientos de lideraz- go transaccional en el sector floricultor}

No se encontró evidencia de los dos primeros comportamientos del estilo de liderazgo transaccional (Laissez-Faire y gerencia por excepción pasiva) en los directivos del sector floricultor y en cambio sí se evidenciaron los dos comportamientos más efectivos de este estilo (gerencia por excepción activa y recompensa contingente).

Con relación a la gerencia por excepción activa, se aprecia en las entrevistas realizadas que hay autonomía: "existe empoderamiento de los trabajadores". También se evidenciaron acciones correctivas de los directivos ya que "informan con claridad qué es lo que se espera de ellos -los trabajadores-", "se realizan reuniones para identificar las causas del no cumplimiento del reglamento y dan otra oportunidad". Y cuando no se cumple una meta: "retoman la situación, la analizan en grupo y entre todos localizan la razón por la cual se falló, más que castigar a los responsables, toman una actitud de reflexión sobre puntos negativos, dan otra oportunidad con acompañamiento y plazo para mejorar".

En cuanto a la recompensa contingente, se encontró que los directivos reconocen socialmente los esfuerzos del trabajador, "se fomenta el trabajo en equipo y se crea la necesidad de trabajar así a través de medios motivadores (...)". Sin embargo, no se logró identificar con la información existente cómo se presentan las recompensas en estas empresas.

En resumen, en las empresas floricultoras se evidencian los dos comportamientos más efectivos del liderazgo transaccional (gerencia activa y recompensa contingente), los cuales funcionan más como un espacio de transición hacia un estilo de liderazgo más transformacional. 


\subsection{Dimensiones de liderazgo trans- formacional en el sector floricultor}

El liderazgo transformacional, se caracteriza por el reconocimiento al otro, por la preocupación genuina del líder, por el crecimiento de sus colaboradores (Bass y Steidlmeier, 1999). Con relación a las cuatro dimensiones del liderazgo transformacional, se encontraron comportamientos representativos en cada una con un mayor énfasis en las dimensiones que representa un modelo que en las que hay una mayor interacción con los trabajadores. A continuación se presentan los hallazgos según cada dimensión.

La influencia idealizada que ejercen los líderes se pudo evidenciar en las organizaciones floriculturas en la identificación que muestran los trabajadores con el pensamiento de sus líderes. Se apreció que existe consenso entre lo que piensa el gerente general y lo que piensan sus trabajadores. En las entrevistas se encontró que los gerentes "están con la gente, dan ejemplo", "es una persona con poder de la palabra y de convencimiento; es persistente y genera confianza".

La dimensión de motivación inspiradora, se evidenció en la forma positiva como los trabajadores perciben la orientación y acompañamiento de sus jefes. Los trabajadores entrevistados, reconocieron que "es una persona enfocada en las personas", "sabe delegar", "realizan reuniones mensuales con comités que son elegidos democráticamente para retroalimentar y escuchar inquietudes generales". También, reconocieron que sus directivos los tienen en cuenta porque hay decisiones donde los gerentes buscan la opinión de los colaboradores. Por su parte, los gerentes resaltaron que sus trabajadores respondían positivamente a este trato, ya que "las personas se comprometen y van más allá de cumplir los objetivos básicos". Por otro lado, los gerentes manifestaron preferencia por los trabajadores que "aportan por encima de las funciones establecidas, de los roles y no en términos de tiempo. Aportan ideas, sugieren estrategias (...) es proactivo, dinámico", sugiriendo un interés y forma de trabajar que estimula el ir más allá de lo esperado.
Por la manera como se desarrolló el estudio, no hay evidencia concluyente que permita determinar con certeza el papel del estímulo intelectual en los directivos del sector floricultor, pues si bien se puede inferir que los gerentes son abiertos a tener en cuenta las ideas de los trabajadores, lo cierto es que más allá del reconocimiento de los trabajadores "desarrollan talentos", "realizan una rotación del personal por las diferentes áreas", no se puede evidenciar qué tanto se promueven y se aceptan las nuevas ideas y si en ello se toma en cuenta la capacidad individual para posteriores procesos de crecimiento al interior de las empresas.

La dimensión de consideración individualizada, se evidenció en la forma en que los directivos manejan los correctivos con sus trabajadores. Cuando un trabajador de manera reiterada comete un error, ya que reconocen que "-si la razón- es cuestión de actitud, -los gerentesle dan oportunidad para que reflexione con respecto al sector, indagan sobre su sueño y lo guían a que busque el espacio adecuado". Sin embargo, de manera similar a la anterior dimensión, por la forma en que se realizó el estudio, no hay evidencia concluyente sobre si dicho comportamiento se presenta como política de desarrollo de los trabajadores, como parte del trabajo rutinario, o si es único ante dichos impases.

Es claro que el liderazgo transformacional se manifiesta parcialmente en las empresas del sector floricultor, ya que este estilo resulta evidente en dimensiones como la motivación inspiradora y la influencia idealizada, pero es apenas incipiente en las demás dimensiones. Se logró evidenciar que los directivos son exigentes con los colaboradores y que permiten que estos desarrollen sus labores con un alto grado de autonomía, sin embargo no es evidente, al menos no de manera explícita, que se presenten oportunidades de crecimiento personalizadas.

Una vez analizados los comportamientos de liderazgo transaccional y transformacional y sus dimensiones, a continuación se presenta el análisis de las diez competencias de liderazgo en los líderes del sector floricultor. 


\subsection{Las diez competencias del Diri- gente-Líder en el sector floricultor}

Con base en el marco de las diez competencias del Dirigente-Líder (Páez et al., 2012), se analizó su presencia

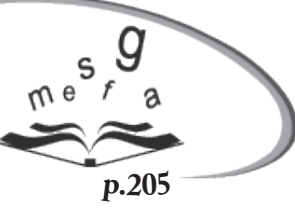

e importancia para cada uno de los grupos estudiados (directivos, personal administrativo y trabajadores), con el objetivo de identificar cuáles competencias predominan por grupo y cómo se relacionan con el liderazgo transformacional.
Integridad y confianza. Se encontró que este es el aspecto más recurrente en las entrevistas a los directivos, pues el $50 \%$ le dio una importancia alta a esta competencia, mientras que para el personal administrativo y los trabajadores, el nivel de preferencia fue del $37,50 \%$ y $43,75 \%$, respectivamente (Ver figura 1). Es así como la integridad y confianza se evidencia como una de las competencias más citadas y de mayor valor para estos grupos, dejando así implícita la importancia de este componente para las organizaciones del sector floricultor.

Figura 1. Importancia de las competencias por grupo organizacional.

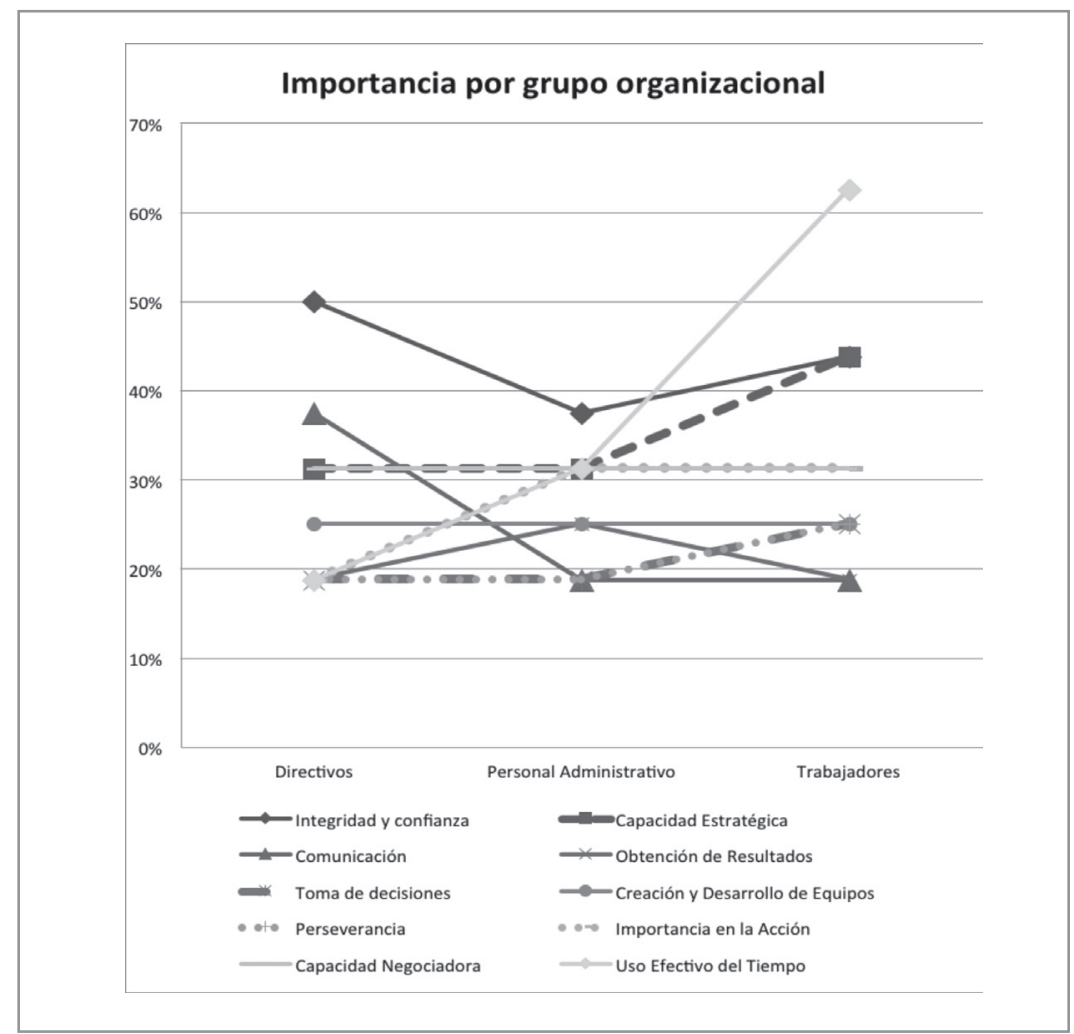

Fuente. Elaboración propia del autor. 
Orientación a la obtención de resultado. Para los directivos, esta competencia fue valorada por el 18,75\%, siendo así una de las competencias con menor preferencia para dicho grupo. Tal comportamiento también se reflejó en el grupo de los trabajadores (18,75\%), el porcentaje más bajo para este grupo. Por su parte, el personal administrativo le dio una preferencia del $25 \%$, que si bien no es la más alta, tampoco es la más baja, lo que evidencia una importancia media para este grupo.

Importancia en la acción. Esta competencia, entendida como la capacidad del líder de convertir en acciones aquellas ideas que se generan en el día a día, sin que sea una necesidad implícita en los largos procesos de planeación, para los directivos tuvo una favorabilidad baja $(18,75 \%)$; mientras que para el resto del personal, tanto trabajadores como personal administrativo, su importancia fue considerada media-alta, con una relevancia evidente de 31,25\%.

Perseverancia. Para los directivos de las organizaciones estudiadas, fue un aspecto sin mucha favorabilidad, pues en las entrevistas realizadas, solo el $18,75 \%$ le dio importancia alta. Igual sucedió con el personal administrativo, mientras que en el grupo de los trabajadores, el $25 \%$ valoró esta competencia como importante.

Uso efectivo del tiempo. Con relación a esta competencia, se encontró un fenómeno interesante y fue que a pesar de las presiones que tienen los directivos, solo el $18,75 \%$ valoró esta competencia como importante, mientras que en los trabajadores resultó ser una de las más importantes (el 62,5\% la evaluó como prioritaria). Esto posiblemente se da por su tipo de trabajo y la forma como laboran en la organización, donde lo más importante para los trabajadores es realizar más, en el menor tiempo posible. Por su parte, el grupo de personal administrativo reflejó un interés medio, con un 31,25\% valorándola como de mucha importancia.

Comunicación. Esta competencia fue la segunda más importante para los directivos después de integridad y confianza, ya que el 37,50\% la favorecieron. Para el personal administrativo y los trabajadores, su relevancia fue una de las más bajas con una preferencia de 18,75\%.

Conformación y desarrollo de equipos de alto desempeño. Esta fue una de las competencias más homogéneas encontradas entre los directivos, el personal administrativo y los trabajadores, ya que todos le dieron una favorabilidad del $25 \%$, lo que refleja coherencia y reconocimiento a la importancia de no solo elegir los colaboradores ideales para un proyecto determinado, sino desarrollarlos.

Toma de decisiones. Para los directivos y el personal administrativo, su favorabilidad fue baja, pues se encuentra en el grupo de aquellas competencias que solo fueron favorecidas por el $18,75 \%$. Para el caso de los trabajadores, el $25 \%$ valoró esta competencia como importante.

Capacidad negociadora. De manera similar a lo ocurrido con la competencia de conformación y desarrollo de equipos de alto desempeño, se encontró una valoración similar para los tres grupos (31,25\%).

Capacidad de direccionamiento estratégico. Para los directivos y el personal administrativo, esta competencia estuvo en el grupo donde se ubican aquellas competencias de favorabilidad media con 31,25\%. Para los trabajadores, fue la segunda competencia más importante con una favorabilidad del $43,75 \%$, solo detrás de la administración del tiempo. 


\section{CONCLUSIONES}

L a principal conclusión de este estudio de casos en el sector floricultor de Colombia, es que el liderazgo transformacional se manifiesta en estos directivos a través de dos dimensiones: influencia idealizada y motivación inspiradora. También, se encontró que estos se caracterizan por desempeñar dos comportamientos de liderazgo transaccional, que son: gerencia activa y recompensa contingente. Este hallazgo resulta alentador, ya que indica que estos diri-gentes cuentan con una base sólida en su liderazgo debido a que tienen dos de las dimensiones de liderazgo transaccional y con los comportamientos transaccionales que han sido considerados como los más efectivos (Bass, 1998). El trasfondo del liderazgo transformacional está en potenciar estilos de liderazgo que ya son efectivos al integrar una mayor sensibilidad humana y sentido de propósito, lo cual deriva en un desempeño superior de su grupo de trabajo al elevar el compromiso y satisfacción de los colaboradores. En otras palabras, lo que muestra este estudio, es que en el sector floricultor de Colombia, existen las bases de un estilo (el liderazgo transformacional) que ha demostrado a nivel mundial ser estimulante, generador de confianza y un motor de desarrollo y crecimiento que genera resultados positivos a nivel individual y organizacional. Se cree que solo con conocer más sobre este enfoque y tomar consciencia sobre las características y bondades del liderazgo transformacional, les ayudaría a los directivos a potenciar su estilo propio, ya que tienen los cimientos para su florecimiento.

En cuanto a las competencias de liderazgo, logró evidenciarse que en los tres niveles organizacionales coincide la importancia que se le da a la integridad y confianza en el liderazgo de estas empresas. Se observa también una gran convergencia en las competencias: capacidad negociadora y creación y desarrollo de equipos. Es importante destacar la diferencia en importancia que se le da a la competencia de comunicación dependiendo del grupo, ya que si bien es de importancia significativa para los directivos y va muy acorde con su rol, para los demás grupos no resulta tan relevante. Es decir, la competencia comunicativa parece ser mucho más valorada por los directivos que por sus colaboradores. En línea inversa, se observa que el uso efectivo del tiempo es fundamental para los trabajadores (quizás debido al enfoque de sus contratos laborales) y en menor medida para el grupo administrativo, para los directivos no tiene mucha relevancia. Llama un poco la atención este aspecto, ya que si bien es cierto que los trabajadores deben cumplir unos horarios y procesos de producción estrictos, los directivos deben enfrentar presiones de mercado y producción global muy exigentes. Por otro lado, llama la atención los resultados con relación a la toma de decisiones, pues se esperaría que los directivos, en quienes está la mayor responsabilidad por procesos que van mucho más allá de la producción, valoraran más esta competencia, que los trabajadores quienes usualmente reciben órdenes y cumplen su parte del proceso. Una posible explicación puede darse en que al formar parte de su rol y su actividad diaria, los ejecutivos no la ven como algo especial sino como una habilidad cotidiana que todos tienen. Sin embargo, este es un aspecto del que deben tomar consciencia, ya que según resultados de estudios realizados por Marcum, Smith y Khalsa (2003), se ha encontrado que aunque el $90 \%$ de estos ejecutivos está completamente seguro de su capacidad para tomar decisiones, más del $50 \%$ de las decisiones de importantes ejecutivos a nivel mundial, fracasan.

El compendio de las competencias utilizadas en este estudio, constituye la personificación más aproximada al ideal del liderazgo en la empresa. Los resultados generales evidencian que independiente del cargo al interior de las empresas floricultoras, las competencias más importantes son integridad y confianza, así como la capacidad negociadora. Ello dice mucho de la cultura organizacional de dichas empresas, que muestran una tendencia a aproximarse más a un estilo de liderazgo transformacional, donde se comienzan a apreciar, en mayor o menor medida las dimensiones propuestas por 
Bass (1998), donde los valores del líder juegan un papel primordial en la creación de confianza, motivación de los trabajadores y estímulos de crecimiento.

Se puede apreciar con base en los resultados ana-lizados, que gran parte de los cimientos para un liderazgo transformacional efectivo, están construidos en estas empresas. Sin embargo, es importante tomar consciencia de ello y crear una estructura que permita desarrollar de una manera explícita, las cuatro dimensiones en conjunto y su aplicación en cada una de las competencias. De esta manera, podrán comenzar a percibir mejor los beneficios que se desprenden de un liderazgo efectivo desde el estilo de liderazgo transformacional. Un curso de investigación futura, puede darse en indagar qué tanto se extienden estos hallazgos a todo el sector floricultor incluidas las empresas que no tienen sello Florverde. También, es importante para las empresas del sector de las flores, que comiencen a desarrollar las dimensiones que no ocupan un lugar destacado en la cotidianidad de sus labores, resulta clave fomentar el pensamiento innovador entre los trabajadores y tener en cuenta las mediciones que se realicen frente a rotaciones de cargos, pues de allí se puede obtener información valiosa sobre las capacidades de las personas que laboran al interior de la empresa. El confiar y tener en cuenta las necesidades individuales permite que exista mayor armonía, lo que se traduce en metas más integradas que desembocan en mayor desempeño y satisfacción general. 


\section{REFERENCIAS BIBLIOGRÁFICAS}

Álvarez de Mon et al. (2001). Paradigmas del liderazgo. Claves de la dirección de personas. Primera edición. McGrawHil. España.

Antonakis, J. y House, R. (2002). The full range leadership theory: The way forward. Ciudad: Editorial.

Avolio, B. Walumbwa, F., y Weber, T. (2009). Leadership: Current theories, research, and future directions. Annual Review of Psychology, 60(1), 421-449.

Avolio, B. Zhu, W., Koh, W., y Bhatia, P, (2004). Transformational leadership and organizational commitment: Mediating role of psychological empowerment and moderating role of structural distance. Journal of Organizational Behavior, 25(8),951-968.

Bandura, A. (1986). Social foundations of thought and action. Englewood Cliffs, NJ: Prentice Hall.

Barling, J. Christie, A., y Turner, N. (2008). Pseudo-transformational leadership: Towards the development and test of a model. Journal or Business Ethics, 81(4), 851-861.

Barling, J. Weber, T., y Kelloway, K. (1996). Effects of transformational leadership training on attitudinal and financial outcomes: A field experiment. Journal of Applied Psychology, 81(6), 827-832.

Bass, B. (1998). Transformational leadership: Industry, military, and educational impact. Mahwah, NJ: Erlbaum.

Bass, B. (1999). From Transactional to Transformational Leadership: Learning to Share the Vision. Binghamton, USA: Editorial.

Bass, B. (1985). Leadership and performance beyond expectations. New York: Free Press.

Bass, B. Avolio, B., Jung, D., y Berson, Y. (2003). Predicting unit performance by assessing transformational and transactional leadership. Journal of Applied Psychology, 88(2), 207-218.

Bass, B. y Steidlmeier, P. (1999). Ethics, character, and authentic transformational leadership behavior. Leadership Quarterly, 10(2), 181-217.

Berson, J. y Avolio, B., (2004). Transformational leadership and the dissemination of organizational goals: A case study of a telecommunication firm. Leadership Quarterly, October 15(5), 625-646.

Blake, R. y Mouton, J. (1982). Management by grid principles or situationalism: Which? Group and Organization Studies, 7(2), 207-210.

Bono, J. y Judge, T. (2003). Self-concordance at work: Toward understanding the motivational effects of transformational leaders. Academy of Management Journal, October 46(5), 554-571. 
Brown, M., Treviño, L. y Harrison, D. (2005). Ethical leadership: A social learning perspective for construct development and testing. Organizational Behavior and Human Decision Processes, 97(2), 117-134.

Burns, J.M. (1978). Leadership. New York: Harper \& Row.

Carl, D. y Javidan, M. (2001). Universality of charismatic leadership: A multi-nation study, paper presented at the National. Academy of Management Conference, Washington.

Carson, J., Tesluk, P. y Marrone, J. (2007). Shared leadership in teams: An investigation of antecedent conditions and performance. Academy of Management Journal, 50(5), 1217-1234.

Ciulla, J. (1998). Ethics, the heart of leadership. Westport, CT: Praeger Publishers.

Colbert, A., Kristof-Brown, A., Bradley, B. y Barrik, M. (2008). CEO Transformational Leadership: The Role of Goal Importance Congruence in Top Management Teams. Academy of Management Journal 51(1), 81-96.

Dirks, K. y Ferrin, D. (2002). Trust in Leadership: Meta-Analytic Findings and Implications for Research and Practice. Journal of Applied Psychology, 87(4), 611-628.

Dvir,T., Kass, N. y Shamir, B., (2004). The emotional bond: Vision and organizational commitment among high-tech employees. Journal of Organizational Change Management, 17(2), 126-143.

Eagly, H., Johannesen-Schmidt, M. y Van Engen, M. (2003). Transformational, transactional, and laissez-faire leadership styles: A meta-analysis comparing woman and men. Phsychological Bulletin 129(4), 569-591.

Eisenbeiss, S., Knippenberg, D. y Boerner, S. (2008). Transformational leadership and team innovation: Integrating team climate principles. Journal of Applied Psychology 93(6), 1438-1446.

García, V., Lloréns, F. y Verdú, A. (2008). The effects of transformational leadership on organizational performance through knowledge and innovation. British Journal of Management 19(4), 299-313.

Gini, A. (1998). Moral leadership and business ethics. In J. Ciulla (Ed.), Ethics, the heart of leadership (pp.27-45). USA: Praeger Publishers.

Graen, G. y Uhl-Bien, M. (1995). Relationship-based approach to leadership: Development of leader-member exchange $(L M X)$ theory of leadership over 25 years: Applying a multi-domain perspective. Leadership Quarterly, 6(2), 219-247.

Hinkin, T. y Schriescheim, C. (2008). An examination of 'nonleadership': From laissez-faire leadership to leader reward omission and punishment omission. Journal of Applied Psychology, 93(6), 1234-1248.

Hoffman, B., Bynum, B., Piccolo, R., y Sutton, A. (2011). Person-organization value congruence: How transformational leaders influence work group effectiveness. Academy of Management Journal, 54(4), 779-796.

Holloway, J. (2012). Leadership Behavior and organizational climate: An empirical study in a non-profit organization, Emerging Leadership Journeys, 5(1), 9-35. 
House, R., Javidan, M., Hanges, P. y Dorfman, P. (2002). Understanding cultures and implicit leadership theories across the globe: An introduction to project GLOBE. Journal of World Business, 37(1), 3-10.

Judge, T. y Piccolo, R. (2004). Transformational and transactional leadership: A meta-analytic test of their relative validity. Journal of Applied Psychology, 89(5), 755-768.

Knights, D. y O'Leary, M. (2006). Leadership, ethics and responsibility to the other. Journal of Business Ethics, 67(2), 125-137.

Kort, E. (2008). What, after all, is leadership? 'Leadership' and plural action. The Leadership Quarterly, 19(4), 409-425.

Lepine, J., Piccolo, R., Jackson, C., Mathieu, J. y Saul, J. (2008). A meta-analysis of teamwork processes: Tests of a multidimensional model and relationships with team effectiveness criteria. Personnel Psychology, 61(2), 273307.

Lewicki, R., Saunders, D. y Minton, J. (1999). Negotiation: Readings, exercises, and cases (3rd ed.). Boston, MA: McGraw-Hill.

Liden, R., Sparrowe, R. y Wayne, S. (1997). Leader member exchange theory: The past and potential for the future. In G. R. Ferris \& K. M. Rowland (Eds.), Research in personnel and human resources management, 15, 47-119. Greenwich, CT: JAI Press.

Ling, Y., Simsek, M., Lubatkin, H. y Veiga, J. (2008). Transformational Leadership's Role in Promoting Corporate Entrepreneurship: Examining the CEO-TMT Interface. Academy of Management Journal 51(3), 557-576.

Liu, J., Siu, O. y Shi, K., (2010). Transformational leadership and employee well-being: the mediating role of trust in the leader and self-efficacy. Applied Psychology: An International Review, 59(3), 454-479.

Lorinkova, N., Pearsall, M. y Sims, H. Jr. (2013). Examining the differential longitudinal performance of directive versus empowering leadership in teams. Academy of Management Journal, 56(2), 573-596.

Lowe, K., Kroeck, K. y Sivasubramaniam, N. (1996). Effectiveness correlates of transformational and transactional leadership: A meta-analytic review of the MLQ literature. Leadership Quarterly, 7(3), 385-425.

Luecke (2003). Negotiation. Harvard Business School Publishing, Boston.

Marcum, D. Smith, S. y Khalsa, M. (2003). Business think: Principios para tomar decisiones acertadas en los negocios. New York: John Willey \& Sons.

Messick, D., y Bazerman, M. (1996). Ethical leadership and the psychology of decision making. Sloan Management Review(Winter), 9-22.

Páez, I., González, A., Enciso, E., Perilla, L. y Martínez, M. (2012). Diez competencias del dirigente líder. Un marco conceptual desde el enfoque del liderazgo transformacional. Bogotá: Universidad Externado de Colombia.

Páez, I., Maldonado, V. y Murillo, C. (2004). Avances: Competencias para el Liderazgo Gerencial. Avances de Liderazgo Nro. 1. Bogotá: Universidad Externado de Colombia. 
Podsakoff, P., MacKenzie, S., Moorman, R. y Fetter, R. (1990). Transformational leader behaviors and their effects on followers' trust in leader, satisfaction, and organizational citizenship behaviors. Leadership Quarterly, 1(2), 107142.

Rowe G. y Nejad M. (2009). Strategic leadership: short-term stability and long-term viability. Ivey Business Journal, 73(5), 2-6.

Schaubroeck, J., Lam, S. y Cha, S. (2007). Embracing transformational leadership: team values and the impact of leader behavior on team performance. Journal of Applied Psychology, 92(4), 1020-1030.

Schriesheim, C., Castro, S., Zhou, T. y DeChurch, L. (2006). An investigation of path-goal and transformational leadership theory predictions at the individual level of analysis. Leadership Quarterly, 7(1), 323-352.

Shamir B., House R. y Arthur, M. (1993). The motivational effects of charismatic leadership: a self-concept based theory. Organizational Science, 4(4), 577-594.

Shin, J. y Zhou, J. (2003). Transformational leadership, conservation, and creativity: Evidence from Korea. Academy of Management Journal, 46(6), 703-714.

Simons, T. (1999). Behavioral integrity as a critical ingredient for transformational leadership. Journal of Organizational Change Management, 12(2), 89-104.

Walumbwa, F., Avolio, B., Gardner, W., Wernsing, T., y Peterson, S. (2008). Authentic leadership: Development and validation of a theory-based measure. Journal of Management, 34(1), 89-126.

Walumbwa, F., Avolio, B., Zhu, W., (2008). How transformational leadership weaves its influence on individual job performance: the role of identification and efficacy beliefs. Personnel Psychology, 61(4), 793-825.

Wang, G., Oh, I., Courtright, S. y Colbert, A., (2011). Transformational leadership and performance across criteria and levels: A meta-analytic review of 25 years of research. Group and Organization Management, 36(2), 223-270.

Yammarino, F., Dionne, S., Chun, J. y Dansereau, F. (2005). Leadership and levels of analysis: A state-of-the-science review. The Leadership Quarterly, 16(6), 879- 919.

Yukl, G. (2012). Leadership in Organizations. 8th Ed. Upper Saddle River, NJ: Pearson/Prentice Hall.

Yukl, G. y Van Fleet, D.D. (1992). Theory and research on leadership in organizations. En D. Dunnette y L.M. Hough (Eds.), Handbook of Industrial and Organizational Psychology, (2nd ed., Vol.3, pp.147-198). Palo Alto, CA: Consulting Psychologist Press.

Zhang, X. y Bartol, K. (2010). Linking empowering leadership and employee creativity: The influence of psychological empowerment, intrinsic motivation, and creative process engagement. Academy of Management Journal, 53(1), 107-128.

Zohar, D. y Tenner-Gazit, O. (2008). Transformational leadership and group interaction as climate antecedents: A social network analysis. Journal of Applied Psychology 93(4), 744-757. 